\title{
On Relation between Similarity Ratio and Acoustic Damping Ratio in One Dimensional Sound Field Partitioned by Perforated Plate with Geometric Similarity
}

\author{
Kunihiko ISHIHARA and Akari GOTO
}

\begin{abstract}
In heat exchangers like a boiler, as a tube bank is set in a duct the alternative vortices occur behind a tube bank and the vortex shedding frequency increases with the flow velocity when the boiler is operated. High level sound is suddenly generated when the vortex frequency comes close to the acoustic natural frequency of the duct. The high level sound keeps the frequency and the amplitude constant even if the flow velocity increases in the case of small acoustic damping due to the self-excited mechanism. This is generally called the self-sustained tone and the factory is forced to stop the operation due to the complaints of the neighborhood. The effective countermeasure is required in the design stage. One of the authors has many studies concerned to the clarification of the generation mechanism and the counter- measures and their effects. In recently, it was clarified that the perforated plate suppressed the high level sound. The author has a question what is the relation between the suppression effect of the perforated plate and the dimension ratio. Then in this paper, after confirming the validity of the analysis we tried to obtain the relation between the acoustic damping ratio and dimension ratio theoretically for several geometrically similar ducts. As a result, it was clarified that the acoustic damping ratio became smaller with larger of dimension ratio.
\end{abstract}

Index Terms - Noise control, Sound and acoustics, Acoustic damping ratio, Perforated plate, Transfer matrix method, Similarity ratio

\section{INTRODUCTION}

In a heat exchanger like a boiler, as a tube bank is set in a duct, the alternative vortices generate in and behind the tube bank when the boiler is operated. The frequency increases with the flow velocity and the high level sound suddenly generated when it gets closer to the natural acoustic frequency of the duct.

This high level sound keeps an air column resonance frequency and the magnitude constant even if increasing the flow velocity in the case of the small acoustic damping [1],[2]. Because the self-exciting mechanism works in the small damping. This phenomenon is called the self-sustained tone and it is often obliged to stop the operation of the factory due

Kunihiko Ishihara, Department of Health and Welfare, Tokushima Bunri University, Shido, Sanuki-city, Kagawa, Japan, +81878997247.

Akari Goto, Department of Health and Welfare, Tokushima Bunri University, Shido, Sanuki-city, Kagawa, Japan, +81878997257. to the complaints of the neighbors. Therefore it is necessary not to be generated such a self-sustained tone in a design stage.

For these problems, Ishihara et al. succeeded to suppress the noise by setting the perforated plate to the duct walls with the cavity [3], [4] and have discussed in detail about the role of the perforated plate in the sound fields [5].

The perforated plates have been used in silencers for a compressor and soundproof walls for the rail way as absorbing materials [6],[7]. There is the detail investigation by Melling about the absorbing action of the perforated plate alone. This was carried out under the condition of high sound pressure of $135 \mathrm{~dB}$ [8]. Melling measured the damping characteristics of the perforated plate by using the one dimensional sound field and compared with the damping element model of the perforated plate proposed by Ingard [9] and Sivian [10].

In during studying these problems, the following question arises how is the relation between acoustic damping and the similarity ratio.

Then one of the authors carried out the acoustic experiment by using two geometrically similar ducts and the acoustic damping ratios were compared [11]. This acoustic damping ratio is the index of evaluating the sound pressure level at resonance. As a result, it was clarified that the acoustic damping ratio became smaller with increasing the similarity ratio. However this experiment was carried out in similarity ratio twice at most. Therefore we can't know the relation between the acoustic damping and the similarity ratio $s$ in the very large $s$ of real thing. Then in this study the relation between the acoustic damping and the similarity ratio will be clarified by the analysis.

\section{OBJECT TO BE CONSIDERED}

Fig. 1 shows the duct system to be considered in this study. The cross section is the square of $100 \mathrm{~mm} \times 100 \mathrm{~mm}$ and the total length is $427 \mathrm{~mm}$ including the thickness of the perforated plate of $10 \mathrm{~mm}$. The diameter of the hole is $2.3 \mathrm{~mm}$ and the number of holes is 96 and the aperture ratio of this plate is $4 \%$.

The frequency analysis is performed for geometrically similar ducts with the similarity ratios of 1,2, 5, 10,20 and 50, and the acoustic damping ratio at each mode is obtained by using the half power method described with Eq. (1) and the resonance curve.

The acoustic damping ratio $\zeta$ can be obtained by the half power method described by the Eq.(1) after obtaining the 


\section{On Relation between Similarity Ratio and Acoustic Damping Ratio in One Dimensional Sound Field Partitioned by Perforated Plate with Geometric Similarity}

frequency responses. Where $f_{\mathrm{n}}$ is the peak frequency and $f_{1}$ and $f_{2}$ are the frequencies where the values are $3 \mathrm{~dB}$ lower than the peak value.

$$
\zeta=\frac{f 2-f 1}{2 f \mathrm{n}}
$$

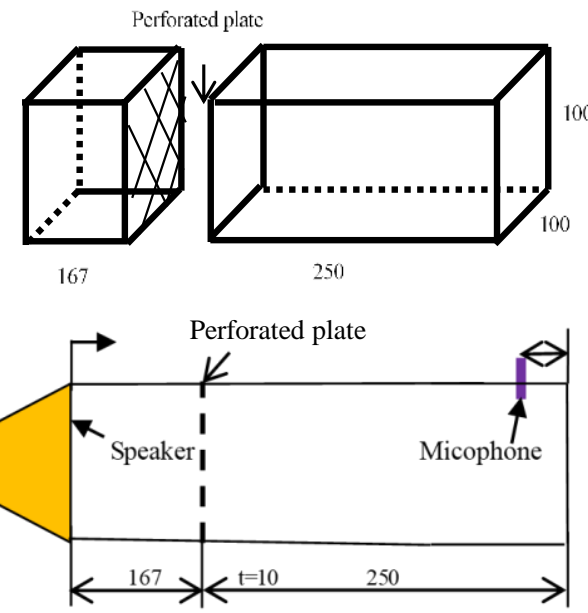

Fig.1 Experimental apparatus

The analytical parameters are the aperture ratio $\varphi$ and the hole length $\mathrm{L}$. The aperture ratios are $1 \%, 2 \%, 5 \%, 10 \%$ and $20 \%$ and the hole lengths are $1 \mathrm{~mm}, 2 \mathrm{~mm}, 5 \mathrm{~mm}, 10 \mathrm{~mm}$ and $20 \mathrm{~mm}$.

\section{ANALYTICAL MODEL AND METHOD}

Fig.2 shows an analysis object duct. The duct is assumed to be one dimensional sound field. Therefore, the frequency range of analyses is limited under $1700 \mathrm{~Hz}$. Because the frequency holding the plane wave is given by $\mathrm{f}=\mathrm{c} / 2 \mathrm{a}$ ( $\mathrm{a}$ : Edge length of square cross section). The calculating frequency result is $f=340 / 0.2=1700 \mathrm{~Hz}$. The numbering is performed as shown in Fig. 2 and the state vector at each point is described by $\left[\begin{array}{ll}P_{i} & U_{i}\end{array}\right]^{T}$. The state vectors at both ends hold the relationship as shown in Eq.(2). Where $P_{\mathrm{i}}, U_{\mathrm{i}}$ are the sound pressure and the volume velocity, respectively.

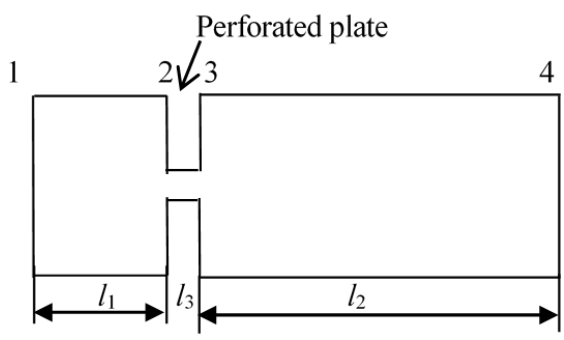

Fig.2 Detail of tube bank part

$$
\begin{aligned}
{\left[\begin{array}{l}
P_{4} \\
U_{4}
\end{array}\right] } & =\left[\begin{array}{cc}
\cos k l_{2} & \mathrm{j} \mathrm{Z}_{2} \sin k l_{2} \\
\frac{1}{\mathrm{Z}} \sin k l_{2} & \cos k l_{2}
\end{array}\right]\left[\begin{array}{cc}
1 & \mathrm{Z}_{\mathrm{R}}+\mathrm{j} \mathrm{Z}_{\mathrm{i}} \\
0 & 1
\end{array}\right]\left[\begin{array}{cc}
\cos k l_{1} & \mathrm{j} \mathrm{Z}_{1} \sin k l_{1} \\
\mathrm{j} \frac{1}{\mathrm{Z}_{1}} \sin k l_{1} & \cos k l_{l}
\end{array}\right]\left[\begin{array}{l}
P_{1} \\
U_{l}
\end{array}\right] \\
& =\left[\begin{array}{ll}
\mathrm{A} 11 & \mathrm{~A} 12 \\
\mathrm{~A} 21 & \mathrm{~A} 22
\end{array}\right]\left[\begin{array}{l}
P_{1} \\
U_{1}
\end{array}\right]
\end{aligned}
$$

Where $Z_{\mathrm{i}}=\rho c / S_{\mathrm{i}}, S_{\mathrm{i}}$ is a cross sectional area. And Aij is the result of multiplication of three matrixes. The aperture ratio $\varphi$ is defined by $S_{3} / S_{1}$, and $Z=z / S$.

Giving the unit forced displacement at the left end of the duct and considering closed at the right end, the boundary conditions are given as follows.

$$
U_{I}=1 \text { and } U_{4}=0
$$

As the applicability range of the acoustic impedance is $r_{0} \sqrt{\omega \rho / \mu}>10$, the corresponding impedance of Melling becomes Eq.(4).

$$
\begin{aligned}
\mathrm{z} & =\left(\frac{2 \mu \mathrm{l}_{3}}{\mathrm{r}_{0}}\right) \sqrt{\frac{\omega \rho}{2 \mu}}(1+\mathrm{j})+j \omega \rho \mathrm{l}_{3}: \\
& =\left(2 \mu \mathrm{l}_{3} / \mathrm{r}_{0}\right) \sqrt{\omega \rho / 2 \mu}+\mathrm{j} \omega \rho \mathrm{l}_{3}\left[1+\frac{1}{\mathrm{r}_{0}} \sqrt{\frac{2 \mu}{\omega \rho}}\right]
\end{aligned}
$$

Where $r_{0}$ is the radius of hole, $l_{3}$ is hole length (plate thickness), $\omega$ is the angular frequency. The air density is $\rho=1.2 \mathrm{~kg} / \mathrm{m}^{3}$ and the viscosity of air is $\mu=1.882 \times 10^{-5} \mathrm{~Pa} \cdot \mathrm{s}$. As a result, A11 A22 of Eq.(2) become as follows.

$\mathrm{A} 11=\cos k l_{2} \cos k l_{1}-\frac{Z_{i}}{Z_{1}} \cos k l_{2} \sin k l_{1}-\frac{Z_{2}}{Z_{1}} \sin k l_{2} \sin k l_{1}+j \frac{Z_{R}}{Z_{1}} \cos k l_{2} \sin k l_{1}$

$\mathrm{A} 12=-Z_{R} \cos k l_{2} \cos k l_{1}-j\left[Z_{1} \cos k l_{2} \sin k l_{1}+Z_{i} \cos k l_{2} \cos k l_{1}+Z_{2} \sin k l_{2} \cos k l_{1}\right]$ $\mathrm{A} 21=\frac{Z_{R}}{Z_{1} Z_{2}} \sin k l_{2} \sin k l_{1}-j\left[\frac{1}{Z_{2}} \sin k l_{2} \cos k l_{1}-\frac{Z_{i}}{Z_{2} Z_{1}} \sin k l_{2} \sin k l_{1}+\frac{1}{Z_{1}} \sin k l_{1} \cos k l_{2}\right]$ $\mathrm{A} 22=\left[\cos k l_{2} \cos k l_{1}-\frac{Z_{1}}{Z_{2}} \sin k l_{2} \sin k l_{1}-\frac{Z_{i}}{Z_{2}} \sin k l_{2} \cos k l_{1}\right]+j \frac{Z_{R}}{Z_{2}} \sin k l_{2} \cos k l_{1}$

Obtaining the sound pressure $\mathrm{P} 4$ of the right end as shown in Fig. 1 by using these equations, Eq. (5) can be obtained as follows.

$$
\mathrm{P} 4=\frac{-\mathrm{A}_{11} \mathrm{~A}_{22}+\mathrm{A}_{12} \mathrm{~A}_{21}}{\mathrm{~A}_{21}}
$$

The frequency response of each case was calculated by use of Eq.(5). And $r_{0}$ was the real hole radius.

\section{ANALYTICAL RESULTS AND COMPARISON WITH EXPERIMENTAL RESULTS}

Here only the acoustic damping ratio of first mode is mentioned because of the first mode being sensitive to the aperture ratio and the hole length.

In the previous study, one of the authors obtained the acoustic damping ratio of first mode for two ducts with geometrically similarity analytically and experimentally and compared the both [11]. Here the duct with similarity ratio 1.0 is called "Duct C" and 2.0 is called "Duct A".

The acoustic damping of only perforated plate $\zeta_{\mathrm{a}}$ is considered in the analysis. On the other hand, the acoustic damping obtained by the experiment $\zeta_{\mathrm{e}}$ is also included the acoustic damping $\zeta_{0}$ in the case of no perforated plate. Therefore $\zeta_{\mathrm{a}}$ should be compared with $\zeta_{\mathrm{e}}-\zeta_{0}$ for comparison between the calculation and the experiment. 
Table1 Acoustic damping ratio of 1st mode for two duct systems in case of without perforated plate (Experiment)

\begin{tabular}{|c|c|}
\hline Kind of duct & $1^{\text {st }}$ mode \\
\hline Duct A & 0.036 \\
\hline Duct C & 0.020 \\
\hline Mean of A and C & 0.028 \\
\hline
\end{tabular}

The acoustic damping in the case of no perforated plate were 0.036 for Duct A and 0.020 for Duct C, respectively. Average of two ducts was 0.028 . Fig. 3 shows the comparison between the analytical result and the experimental result of acoustic damping ratios of two ducts for several cases. Table 2 shows the experimental parameters.

The analytical result is approximately agreement with the experimental result. It can be considered to be able to evaluate the acoustic damping ratio by the analysis.
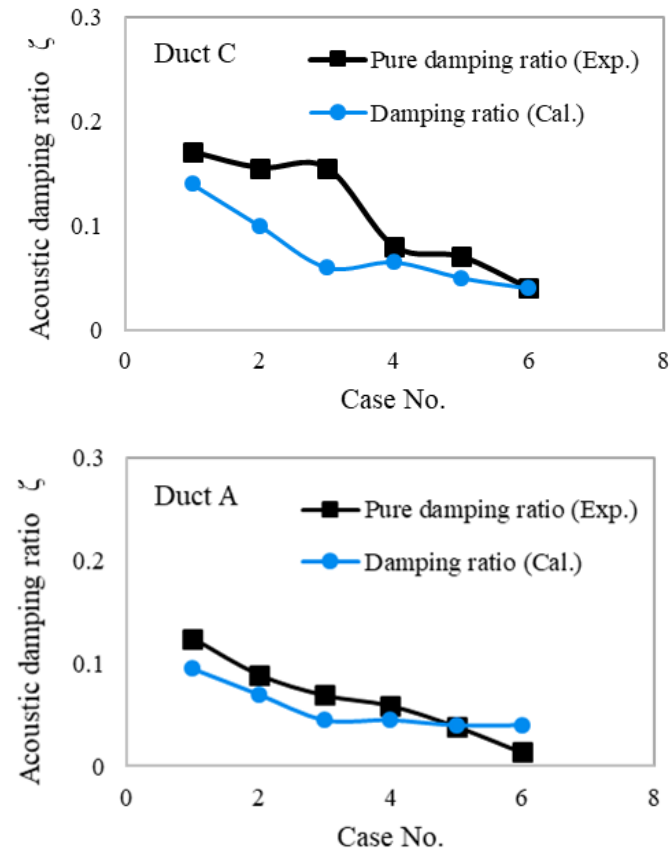

Fig. 3 Comparison of acoustic damping ratio of two ducts

Table 2 Experimental parameters.

\begin{tabular}{|c|c|c|c|}
\hline Kinds of duct & $\begin{array}{c}\text { Hole diameter(mm) } \\
\text { and numbers }\end{array}$ & $\varphi$ & Case No. \\
\hline \multirow{6}{*}{$\begin{array}{l}\text { Duct A } \\
\text { Length: } 834 \mathrm{~mm} \\
\text { Cross } \\
\text { section: } 200 \mathrm{~mm} \times 200 \mathrm{~mm} \\
\text { Similarity ratio: } 2.0\end{array}$} & $2.3 \varphi-100$ & $1 \%$ & 1 \\
\hline & $3.0 \varphi-64$ & $1 \%$ & 2 \\
\hline & $4.5 \varphi-25$ & $1 \%$ & 3 \\
\hline & $2.3 \varphi-400$ & $4 \%$ & 4 \\
\hline & $3.0 \varphi-256$ & $4 \%$ & 5 \\
\hline & $4.5 \varphi-100$ & $4 \%$ & 6 \\
\hline \multirow{6}{*}{$\begin{array}{l}\text { Duct C } \\
\text { Length: } 417 \mathrm{~mm} \\
\text { Cross } \\
\text { section: } 100 \mathrm{~mm} \times 100 \mathrm{~mm} \\
\text { Similarity ratio: } 1.0\end{array}$} & $1.2 \varphi-100$ & $1 \%$ & 1 \\
\hline & $1.5 \varphi-64$ & $1 \%$ & 2 \\
\hline & $2.3 \varphi-25$ & $1 \%$ & 3 \\
\hline & $1.2 \varphi-361$ & $4 \%$ & 4 \\
\hline & $1.5 \varphi-225$ & $4 \%$ & 5 \\
\hline & $2.3 \varphi-100$ & $4 \%$ & 6 \\
\hline
\end{tabular}

\section{V . ANALYTICAL RESULTS AND CONSIDERATIONS}

\section{A. Relation between Similarity Ratio and Sound Pressure Level}

Fig.4 Fig.9 show the frequency response analysis results in the cases of similarity ratio being 1.0, 2.0, 5.0, 10.0, 20.0 and 50.0. In general, the inverse relation exists between the similarity ratio and the natural acoustic frequency. when the maximum frequency in analysis is $1800 \mathrm{~Hz}$ in the similarity ratio of 1.0 the maximum frequencies become $900 \mathrm{~Hz}, 360 \mathrm{~Hz}$, $180 \mathrm{~Hz}, 90 \mathrm{~Hz}$ and $36 \mathrm{~Hz}$ corresponding to the similarity ratio.

The peak sound pressure level at resonance must be caught certainly to obtain the acoustic damping ratio accurately. Therefore it is necessary to make it as small as possible.

Here the frequency resolution was gradually reduced for each duct and the frequency responses did not change, that is to say, the calculation was performed with frequency resolution catching the peak.

The value of frequency indicated in these figures shows the frequency resolution. It can be seen from these figures that the resonance curves become steep with increasing the similarity ratio. This means the acoustic damping becoming smaller. We will present the relation between the similarity ratio and the acoustic damping ratio in the next section.

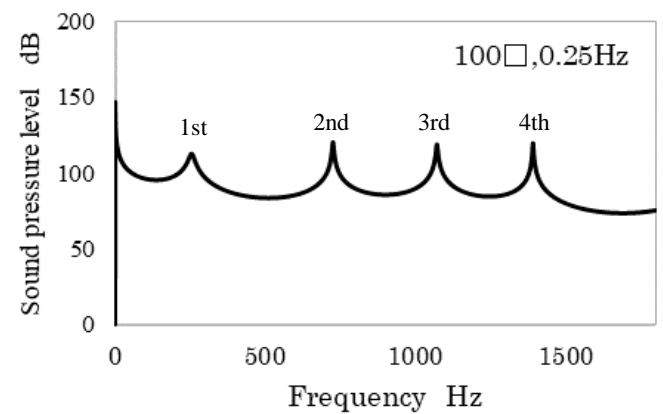

Fig.4 Frequency response of $100 \square$ duct

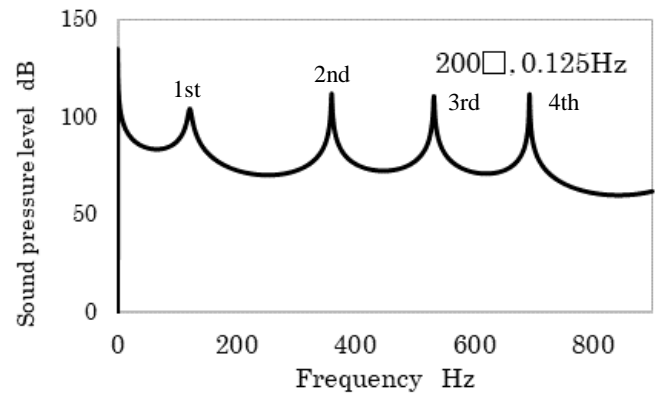

Fig. 5 Frequency response of $200 \square$ duct

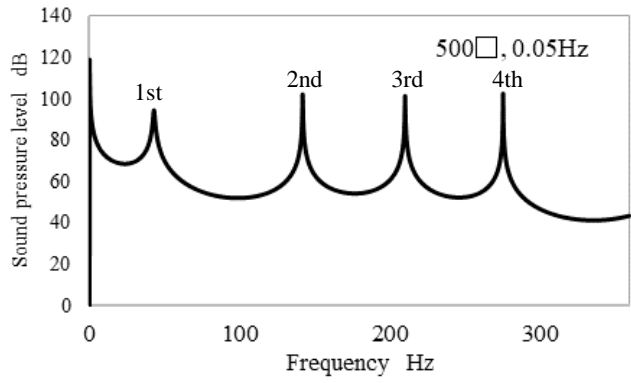

Fig.6 Frequency response of 500 $\square$ duct 


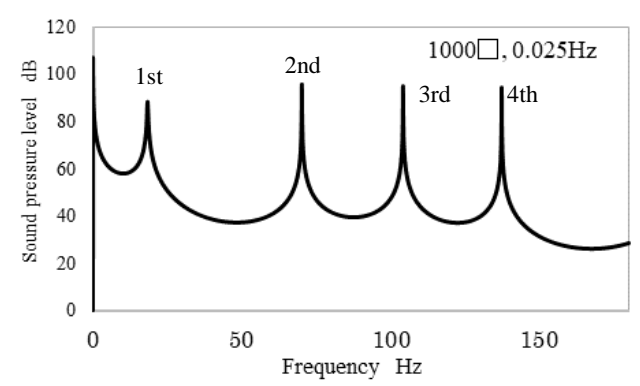

Fig.7 Frequency response of $1000 \square$ duct

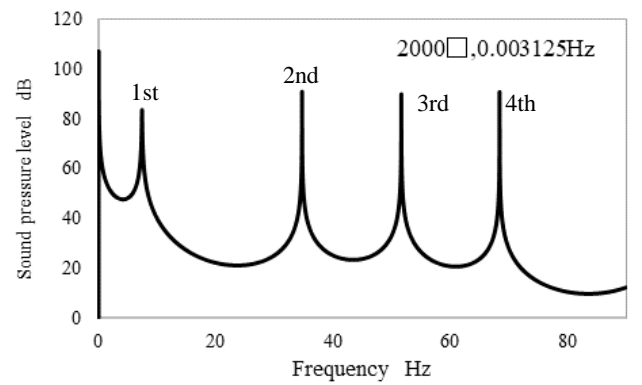

Fig.8 Frequency response of $2000 \square$ duct

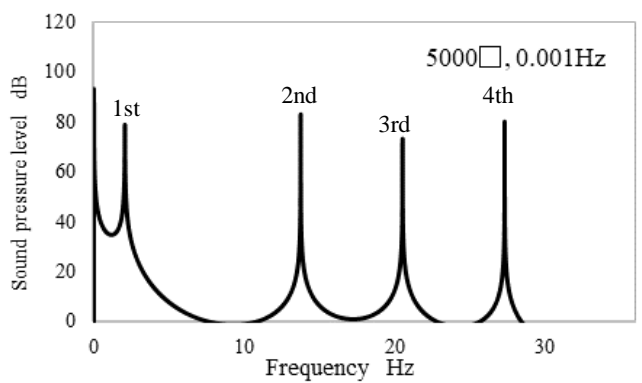

Fig.9 Frequency response of $5000 \square$ duct

\section{B. Relation between Similarity Ratio and Acoustic Damping Ratio}

Table 2 shows the relation between the similarity ratio and the acoustic damping ratio for each mode obtained by the half power method and Fig.10 shows it. As the acoustic damping ratio of $1 \mathrm{st}$ mode is an order of magnitude larger than those of 2nd and 3rd modes, the acoustic damping of 2nd and 3rd are described ten times of those.

From this figure it can be seen that the any acoustic damping ratio becomes smaller with increasing the similarity ratio. The approximate curves are shown by dotted lines and these approximate equations are shown in this figure. From these equations the acoustic damping is proportional to -0.836 $\sim-0.996$ of the similarity ratio. Roughly speaking the acoustic

Table2 Relation between similarity ratio and acoustic damping ratio for each mode

\begin{tabular}{|c|c|c|c|}
\hline Similarity ratio $s$ & $1^{\text {st }}$ mode & $2^{\text {nd }}$ mode & $3^{\text {rd }}$ mode \\
\hline 1.0 & 0.032940 & 0.002760 & 0.002218 \\
\hline 2.0 & 0.020747 & 0.001475 & 0.001116 \\
\hline 5.0 & 0.01153 & 0.000633 & 0.000309 \\
\hline 10.0 & 0.00647 & 0.000214 & $8.4 \mathrm{E}-05$ \\
\hline 20.0 & 0.00334 & $6.31 \mathrm{E}-05$ & $3.63 \mathrm{E}-05$ \\
\hline 50.0 & 0.001285 & $1.09 \mathrm{E}-05$ & $7.32 \mathrm{E}-06$ \\
\hline
\end{tabular}

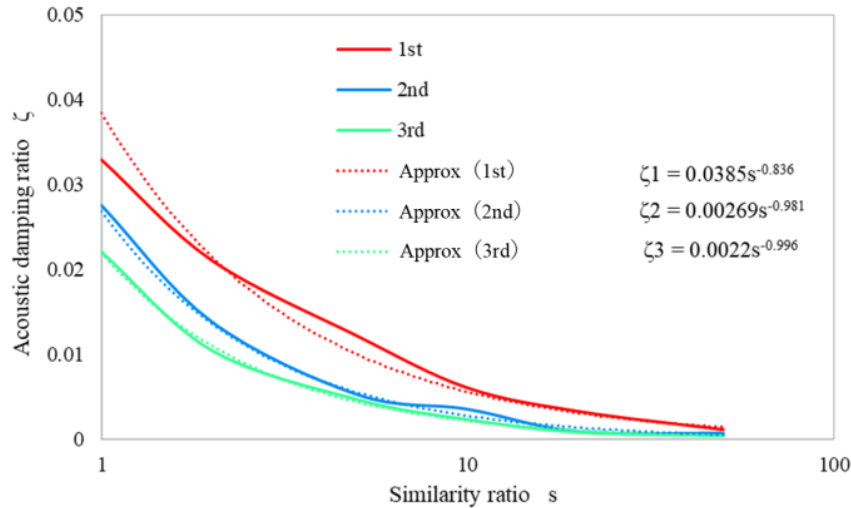

Fig.10 Relation between acoustic damping ratio and similarity ratio

damping seems to be proportional to the reciprocal.

\section{Relation between Similarity Ratio and Natural Acoustic Frequency}

Fig.11 shows the relation between the natural acoustic frequencies of 1 st to $3^{\text {rd }}$ mode and similarity ratio $\mathrm{s}$. The dotted lines are approximate curves and the equations are shown in the figure as $\zeta=a \cdot \mathrm{s}^{\mathrm{b}}$ ( $\mathrm{a}, \mathrm{b}$ are constants). It can be said that the natural acoustic frequency is inversely proportional to the similarity ratio because of $b \fallingdotseq-1.0$. In the case of the acoustic damping ignored completely it can be proved theoretically that the natural acoustic frequency is inversely proportional to the similarity ratio. It is due to the acoustic damping that the value of $b$ is slightly different of -1.0 .

\section{D.Effect of Aperture Ratio on Acoustic Damping}

The acoustic damping ratio of the first mode was calculated for aperture ratios of $1 \%, 2 \%, 5 \%, 10 \%$ and $20 \%$ in order to examine the effect of the aperture ratio on the acoustic damping ratio. The result is shown in Fig.12. It can be seen from this figure that the acoustic damping becomes smaller with the aperture ratio larger. In general, the pressure difference between before and after the perforated plate is larger with the aperture ratio smaller [13]. That is to say, it can be considered the acoustic damping ratio becomes also large as the acoustic resistance becomes large.

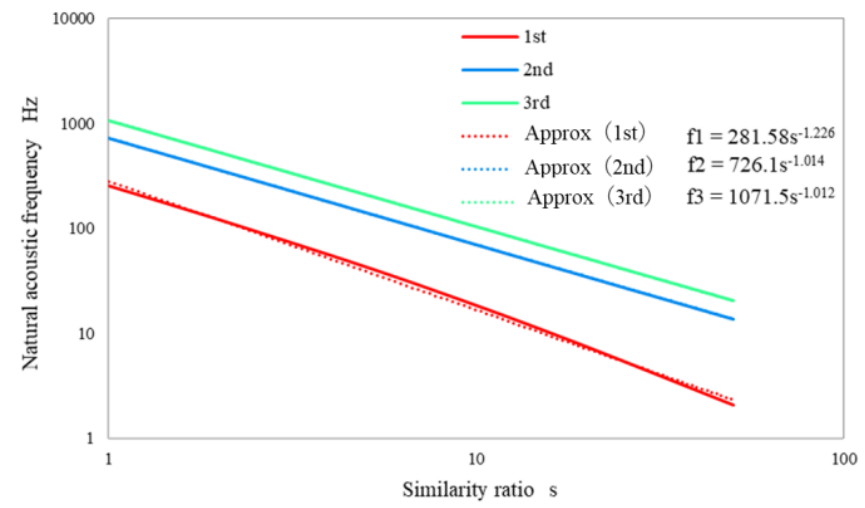

Fig.11 Relation between natural acoustic frequency and similarity ratio 


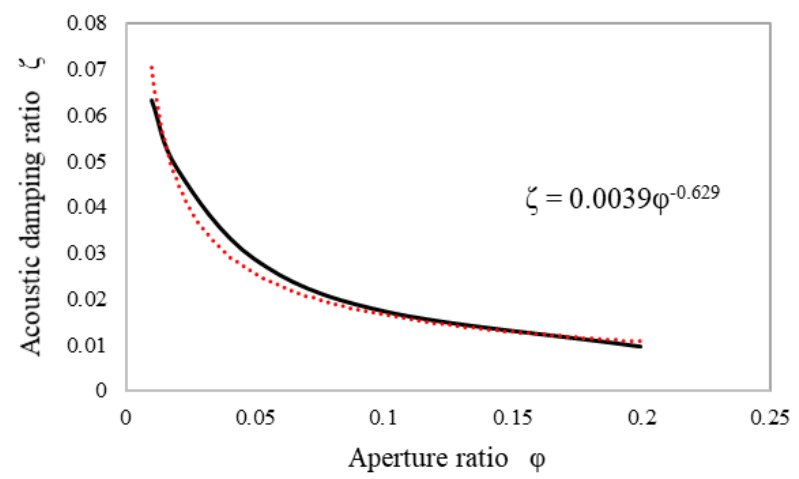

Fig.12 Acoustic damping ratio to aperture ratio

\section{E. Effect of Hole's Length on Acoustic Damping}

The acoustic damping ratio of the first mode was calculated for hole's lengths of $1 \mathrm{~mm}, 2 \mathrm{~mm}, 5 \mathrm{~mm}, 10 \mathrm{~mm}$ and $20 \mathrm{~mm}$ in order to examine the effect of the hole's length on the acoustic damping ratio. The result is shown in Fig.13. It can be seen from this figure that the acoustic damping becomes larger with the hole's length larger. As can be seen from Eq.(4), the real part of the acoustic impedance of the perforated plate is proportional to the hole's length. Therefore it is natural that the acoustic damping ratio becomes larger with the hole's length larger.

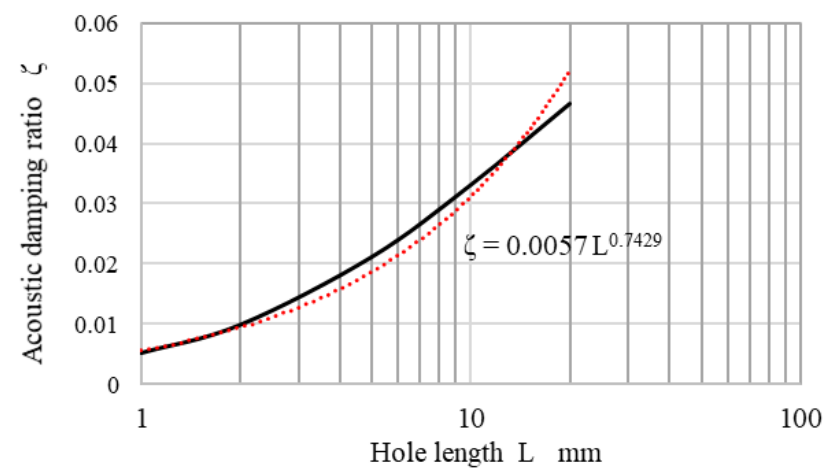

Fig.13 Acoustic damping ratio to hole length

\section{F. Comparison of Acoustic Damping Ratios due to Prediction using Resonance Peak with that of Half Power Method}

It is said that the acoustic damping is inversely proportional to the peak sound pressure at resonance [12]. Therefore, the acoustic damping can be obtained by the peak sound pressure if we can know the acoustic damping and the peak sound pressure at certain condition. Fig.14 (a), (b) show the comparison of the acoustic damping (Blue) by peak sound pressure with that (Red) of the half power method. The both are matched at the point indicated by the circle $\bigcirc$. The both results are good agreement with each other. Therefore, the acoustic damping may be obtained by the peak value. As a result, we can obtain the acoustic damping ratio simply and speedy by using this method.
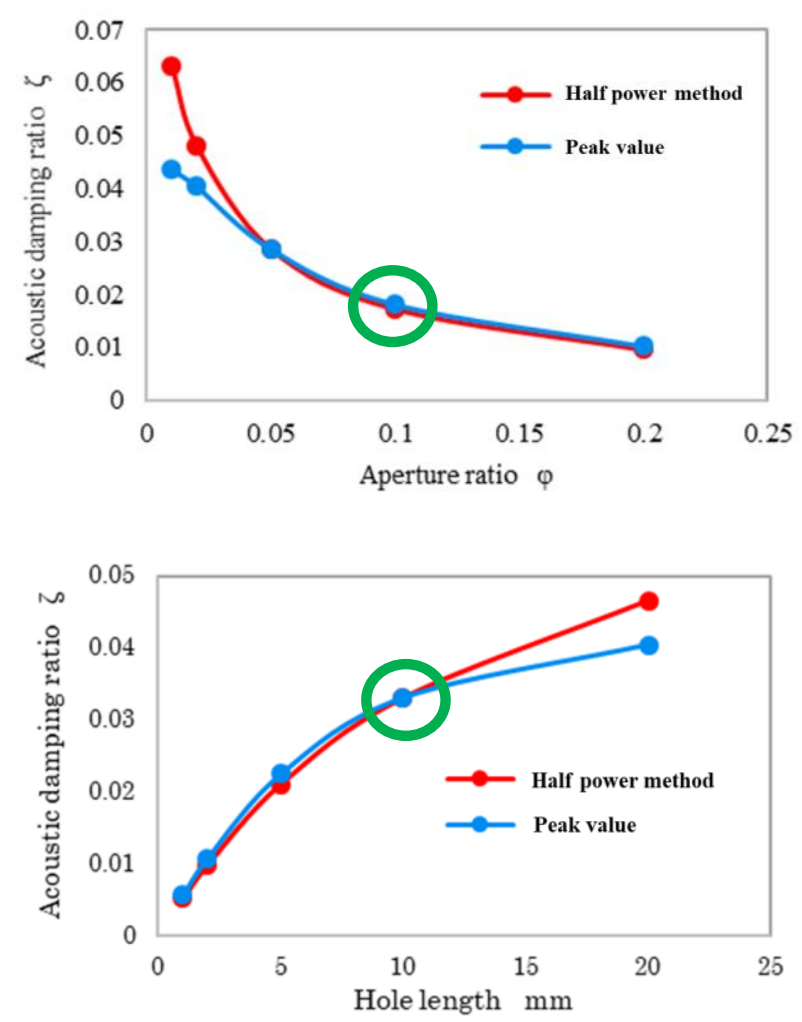

Fig.14 Comparison acoustic damping ratios by half power method with that by peak value

\section{CONCLUSIONS}

The acoustic damping ratio has been examined theoretically and experimentally for one dimensional sound field like a duct with a perforated plate.

This examination was for the similarity ratio 2.0 at most.

However it has not clarified for the similarity ratio $50 \sim 100$ of the real thing.

In this paper, after confirming the applicability of Melling's acoustic impedance we tried to apply it to the analysis of the real thing and also examine the effects of the aperture ratio and the hole's length on the acoustic damping ratio. As a result, the following findings could be obtained.

(1) The acoustic damping ratio $\zeta$ of a geometrically similar duct system becomes smaller with the similarity ratio $\mathrm{s}$ larger. Assuming $\zeta=\mathrm{a} \cdot \mathrm{s}^{\mathrm{b}}$, the values of $\mathrm{b}$ were -0.836 for 1st mode, -0.981 for 2 nd mode and -0.996 for 3rd mode.

(2) The natural acoustic frequency of a geometrically similar duct system is almost proportional to the similarity ratio s.

(3) The acoustic damping becomes larger with the aperture ratio $\varphi$ smaller. Assuming $\zeta=a \cdot \varphi^{b}$, the values of $b$ was -0.629 for 1 st mode.

(4) The acoustic damping becomes larger with the hole's length $\mathrm{L}$ larger. Assuming $\zeta=\mathrm{a} \cdot \mathrm{L}^{\mathrm{b}}$, the values of $\mathrm{b}$ was 0.743 for 1 st mode.

(5) The acoustic damping obtained by the peak sound pressure is good agreement with that by the half power method. It was clarified that the acoustic damping may be obtained by the peak value. It can be done simply and speedy by using this method. 
On Relation between Similarity Ratio and Acoustic Damping Ratio in One Dimensional Sound Field Partitioned by Perforated Plate with Geometric Similarity

\section{REFERENCES}

[1] K, Ishihara, "Study on high SPL sound of gas heater composed of two parallel located ducts with tube bundles (1st Report, Understanding of phenomenon)", Transactions of the Japan Society of Mechanical Engineers, Series B, Vol.70, No.689 (2004a), pp.126-132(in Japanese).

[2] K, Ishihara, "Study on high SPL sound of gas heater composed of two parallel located ducts with tube bundles (2nd Report, Acoustic damping of perforated baffle plate and its effect)", Transactions of the Japan Society of Mechanical Engineers, Series B, Vol.70, No.689 (2004b), pp.133-139(in Japanese).

[3] K, Ishihara, M. Nakaoka and M. Nishioka, "Study on a countermeasure for high level sound generated from boiler tube bank duct using walls made of perforated plate (In case of aperture ratio being more than 1\%)", Transactions of the Japan Society of Mechanical Engineers (in Japanese), Vol.82, No.841 (2016), DOI:10.1299/transjsme.16-00179.

[4] K, Ishihara, "Study on a countermeasure for high level sound generated from boiler tube bank duct using walls made of perforated plate (Grasp critical aperture ratio and influence of cavity volume on suppression effect)", Transactions of the Japan Society of Mechanical Engineers (in Japanese), Vol.83, No.848 (2017), DOI: 10.1299/transjsme. 16-00456.

[5] K, Ishihara, S. Kudo, T. Masumoto and M. Mori, "Study on acoustic natural frequency and its mode of one dimensional sound field partitioned by perforated plate", Transactions of the JSME (in Japanese), Vol.84, No.857 (2018), DOI:10. 1299/transjsme. 17-00365.

[6] Y. Kimura, Z. Yamaguchi and T. Itaya, "Perforated panel type silencer for screw compressors", Kobe Steel Engineering Reports, Vol.59, No.3 (2009), pp.13-16(in Japanese).

[7] K. Tsugihashi, I. Yamagiwa and M. Kikuchi, "Research on Numerical Analysis Technique for Sound Fields including Perforated Plate", Kobe Steel Engineering Report, Vol.64 , No.2(2014), pp.90-95(in Japanese).

[8] T. H. Melling, "The acoustic impedance of perforates at medium and high sound pressure levels", Journal of Sound and Vibration, Vol.29, No.1 (1973), pp.1-65.

[9] U. Ingard, "On the theory and design of acoustic resonators", Journal of Acoustic Society of America, Vol.25(1953), pp.1037-1062

[10] L. J. Sivian, "Acoustic impedance of small orifices". Journal of Acoustic Society of America, Vol.7(1935), pp.94-101

[11] K. Ishihara, "Study on sound pressure level and acoustic damping ratio of one dimensional sound field partitioned by perforated plate with geometric similarity", Transactions of the Japan Society of Mechanical Engineers, Vol.86, No.891, 2020 (in Japanese).

[12] K. Ishihara, A. Goto and M. Kashino, "Relation between peak value and acoustic damping of sound pressure level of one dimensional sound field partitioned with perforated plates", International Journal of Engineering and Applied Science (IJEAS), ISSN:2394-3661, Vol.6, Issue 9, 2019.

[13] K. Ishihara, S. Kudo, T. Masumoto and M. MORI, "Study on acoustic natural frequency and its mode of one dimensional sound field partitioned by perforated plate", Transactions of the Japan Society of Mechanical Engineers, Vol.84, No.857, 2020 (in Japanese).

Kunihiko Ishihara was born in 1947 in Kurashiki City, Okayama Prefecture Japan. He received the B.S. degree from Kobe University in 1969. He got a master's degree in Kobe University in 1971 and earned the Ph.D. degree in Engineering from The Osaka University in 1986.

He worked in Kawasaki Heavy Industry Co. Ltd. as a Mechanical Engineer for 33 years. After that he became a Professor of Tokushima University in 2004. He had been studying the vibration and noise control, above all he studied the flow induced vibration and noise problems. He has authored or co-authored over 100 technical journals and over 50 conference papers. He is a fellow of JSME (Japan Society of Mechanical Engineers) now. He is a Professor of Tokushima Bunri University. He teaches a mechanical field subjects for students.

Akari Goto was born in 1984 in Tonosho, Kagawa Prefecture Japan. She received a bachelor's degree (clinical engineering) from Hiroshima International University in 2007. She got a master's degree in 2009 and earned the Ph.D. degree in Medical Engineering from The Hiroshima International University in 2012.

She became a lecturer at Tokushima Bunri University in 2009. And she has been a teacher since 2011 .
She conducts research on blood coagulation, medical materials, and medical device development. 